\title{
Physical Activity Can Enhance Treatment for Substance Use Disorder: A Systematic Review
}

\author{
Ethan Blake $^{1}$, Antonia Sawyer ${ }^{2}$, Dennis Savaiano ${ }^{3}$ \\ ${ }^{1}$ Indiana University School of Medicine; ${ }^{2}$ Indiana Clinical and Translational Sciences Institute \\ Hoosier Health and Wellness Alliance; ${ }^{3}$ Purdue University Department of Nutrition Science and \\ Indiana Clinical and Translational Sciences Institute Community Health Partnerships
}

Background/Objective: Substance use disorder is a significant yet treatable mental health disorder affecting approximately 20.3 million Americans in 2018. Its continued prevalence indicates the need for additional approaches to complement existing therapies. Physical activity is one potential nontraditional therapy that has been utilized. A qualitative systematic review was conducted to investigate the effects physical activity as a therapy adjunct for non-alcohol and non-tobacco substance use disorder treatment, evaluating effects on substance use and craving levels.

Methods: Using PRISMA guidelines, English language papers in CINAHL, PubMed, Embase, and APA PsycArticles were searched with no date restrictions. In total, 387 abstracts were screened, of which 342 were excluded because they investigated irrelevant outcomes, analyzed solely alcohol or tobacco use disorder, were not primary research, or were duplicates. The remaining 45 citations were reviewed independently by two authors, and 17 were included in the final review. The included studies were graded using a quality criteria checklist based on the quality constructs and domains for research studies reported by the Agency for Healthcare Research and Quality.

Results: Of the 17 included studies, 9 reported favorable outcomes of physical activity on reducing substance use. Four of these articles were of positive quality, and 5 were of neutral quality. Additionally, 4 studies reported favorable outcomes of physical activity on reducing craving levels. All 4 of these studies were of neutral quality. No studies reported unfavorable outcomes.

Conclusion: The findings indicate that exercise may help to reduce substance use and cravings in those undergoing treatment for substance use disorder, but research on which forms of exercise improve treatment for specific substances is needed.

Impact and Implications: This study helps to clarify that further research is needed to optimize the effects of exercise as an adjunct treatment for substance use disorder. 